\title{
A Three-Dimensional Meso-Macroscopic Model for Li-Ion Intercalation
}

\section{Batteries}

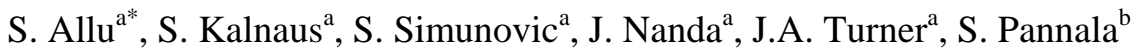 \\ a Oak Ridge National Laboratory, Computer Science and Mathematics Division, Oak Ridge, \\ TN 37831-6164 \\ ${ }^{\text {b }}$ SABIC, Houston, TX
}

\begin{abstract}
In this paper we present a three-dimensional computational formulation for electrode-electrolyteelectrode system of Li-Ion batteries. The physical consistency between electrical, thermal and chemical equations is enforced at each time increment by simultaneously driving the residual of the resulting nonlinear equations to zero. The formulation utilizes a rigorous volume averaging approach typical of multiphase formulation and it was recently extended to modeling of supercapacitors [1]. Unlike the existing battery modeling methods which use segregated solution of conservation equations and idealized geometries, our unified approach can model arbitrary battery and electrode configurations. The consistency of multi-physics solution also allows for consideration of wide array of initial conditions and load cases. The formulation accounts for spatio-temporal variations of material and state properties such as electrode/void volume fractions and anisotropic conductivities. The governing differential equations are discretized using finite element method and solved using non-linear solvers with robust stability and convergence. The new formulation was validated for standard Li-ion cells and compared against experiments. Its scope and ability to capture spatio-temporal variations of potential and lithium distribution were demonstrated on a prototypical three-dimensional electrode problem.
\end{abstract}

\footnotetext{
*Corresponding Author: allus@ornl.gov
} 
Keywords: Li-ion, Modeling and Simulation

\section{Notice}

This manuscript has been authored by UT-Battelle, LLC under Contract No. DE-AC05-00OR22725 with the U.S. Department of Energy. The United States Government retains and the publisher, by accepting the article for publication, acknowledges that the United States Government retains a nonexclusive, paid-up, irrevocable, world-wide license to publish or reproduce the published form of this manuscript, or allow others to do so, for United States Government purposes. The Department of Energy will provide public access to these results of federally sponsored research in accordance with the DOE Public Access Plan(http://energy.gov/downloads/doe-public-access-plan). 


\section{Nomenclature}

\begin{tabular}{|c|c|}
\hline$a_{s}$ & Specific surface area of electrode \\
\hline$c_{e}$ & Concentration of lithium salt in liquid electrolyte phase \\
\hline$c_{s}$ & Surface concentration of lithium in solid phase \\
\hline$c_{s, a v g}$ & Average concentration of lithium in solid phase \\
\hline$c_{s, \max }$ & Maximum concentration of lithium in solid phase \\
\hline$D_{e}$ & Diffusion coefficient of lithium salt in the solution \\
\hline$D_{e}^{e f f}$ & Effective diffusivity of electrolyte \\
\hline$D_{s}$ & Diffusion coefficient of lithium in solid phase \\
\hline$D_{s}^{e f f}$ & Effective diffusivity of solid electrode matrix \\
\hline$f_{+}$ & Activity coefficient of the salt in electrolyte \\
\hline$F$ & Faraday's constant $\left(96485 \mathrm{C} \mathrm{mol}^{-1}\right)$ \\
\hline$i_{0}$ & Exchange current density \\
\hline$j^{L i}$ & Pore wall Li flux across interface \\
\hline$l_{s}$ & Characteristic diffusion length in solid phase \\
\hline$R$ & Universal gas constant $\left(8.3143 \mathrm{~J} \mathrm{~mol}^{-1} \mathrm{~K}^{-1}\right)$ \\
\hline$R_{s}$ & Radius of solid electrode particle \\
\hline$t$ & Time \\
\hline$T$ & Temperature \\
\hline$t_{+}^{0}$ & Lithium cation transference number \\
\hline
\end{tabular}




\begin{tabular}{|ll|}
\hline $\begin{array}{l}U \\
\alpha_{a}\end{array}$ & Equilibrium potential \\
$\alpha_{c}$ & Cathodic transfer coefficient \\
$\varepsilon_{e}$ & Volume fraction of electrolyte \\
$\varepsilon_{s}$ & Volume fraction of solid phase \\
$\varphi_{e}$ & Potential of liquid phase \\
$\varphi_{s}$ & Potential of solid phase \\
$\eta$ & Surface overpotential \\
$\kappa^{e}$ & Ionic conductivity of electrolyte \\
$\kappa^{e f f}$ & Effective ionic conductivity of liquid phase in solid matrix \\
$\kappa_{D}^{e f f}$ & Effective diffusional conductivity of electrolyte \\
& Electric conductivity of solid electrode material \\
\hline
\end{tabular}




\section{Introduction}

Li-Ion battery packs for electric drive vehicles require energy and power densities that can only be achieved by highly energetic materials. Transport limitations of the constituent materials and safe operating conditions of electrode-electrolyte system play an important role to meet the design requirements of these packs. A wide range of cell chemistries and additives has been developed for different applications to improve cell characteristics such as energy/power density [2] and increase safety. New 3D battery architectures [4, 5], both at the cell and the pack level, are under development with a potential to achieve high power and energy densities in smaller footprints compared to the two dimensional counterparts [3]. A physics-based, system level simulation capability can accelerate the battery development by filtering different choices for the electrode materials. In these system-level models one can analyze the effect of various materials and chemistry options, predict system performance, optimize material components and geometry, and address the safety and durability in an integrated fashion [6].

In recent years there has been tremendous advance in research, both experimental and computational, toward improving performance and life of lithium ion batteries. Many causes for capacity loss, namely, lithium deposition, porosity change, film formation, side reactions, thermal abuse, intercalation induced stress generation and mechanical degradation have been identified. Strong improvements of the performance at the cell level have not been accompanied with commensurate improvements at the battery pack level. One of the technologies envisioned to increase battery power and energy densities is the three-dimensional form of battery electrodes. The 3D format brings up additional issues, such as manufacturing feasibility, non-uniform current distribution, stress concentrations, and complex heat dissipation, which need to be analyzed experimentally and computationally. A study of current density distribution in micro batteries 
based on periodic electrode rod elements was presented by R.W.Hart et.al. [7]. Though high capacities are achieved on smaller footprint, many significant issues, such as capacity fade due to local lithium depletion from the electrolyte, remain [8]. It is imperative to understand the interplay among the coupled physicochemical processes (electrical, electrochemical, thermal, mechanical) that span over multiple length scales, and correlate the influence of the microscopic interactions with the macroscopic battery design features for improved battery performance.

Computational models are developed primarily for the lower-length scales (atomistic and mesoscopic) which do not scale to the system level. The existing models at the macroscopic or system-level are primarily based on electrical circuit models or simple 1D models $[9,10,11,12,13]$. Various mathematical formulations, such as the single- particle [14] and porous-electrode models [15], have been employed to estimate the performance of the batteries. In single-particle method, the lithiation state of each electrode is represented by a single particle and thus changes in the concentration of electrolyte across the electrode thickness are neglected. Such simplified approach is applicable to thin electrodes and rather small currents. A one-dimensional model to simulate galvanostatic charge and discharge for a porous electrode and separator system was developed in [15]. The conservation of mass in the solid phase is either explicitly calculated using the Duhamel's superposition principle or solved with a pseudo second dimension i.e., the radius of the particle with rest of the differential equations. Wang et al. [16] presented a relation between the surface concentration to the average concentration of lithium in solid phase to avoid the computationally expensive solution of the solid phase diffusion equation. A comparison of approximate solutions for the solid state diffusion problem can be found in [17].

1D models are limited in their ability to capture spatial variations in permeability or conductivity and cannot handle multidimensional structure of recent electrode and solid electrolyte material. There have been some recent extensions to $2 \mathrm{D}[18,19,20]$ and this is 
still an active area of development. But these models lack the ability to capture nonuniformities in large cells. Recently, a new computational infrastructure for battery simulations [21] has been presented that can loosely couple electrochemical, thermal, and electrical models in three dimensions but the primary transport of ions in the electrochemical models studied was still in one direction. A reformulated porous electrode theory has been developed in [22] using phase field models to describe material transformation consistent with ion intercalation and faradaic kinetics for concentrated solutions. The formulation however is still not three-dimensional as solid diffusion is one dimensional case of idealized spherical geometry.

In this paper, we describe a system-level computational framework that solves the underlying transport and kinetics equations for arbitrary 3D cell configuration as a single domain. In the following section, we present the extension of the porous electrode theory using volume averaging technique that accounts for the spatio-temporal variations at the cell level and is informed by the constitutive models from the lower length scales. In Section 3, we describe the numerical solution method for solving the resulting Differential Algebraic Equation (DAE) system that enforces multi-physics solution consistency. Finally, in Section 4 a series of computational results are presented as verification and validation studies to demonstrate the efficiency of the modeling framework on single domain. A case study of prototype $3 \mathrm{D}$ cell architectures is shown to illustrate the capability of the method.

\section{Continuum electro-chemical model for batteries}

The equations describing the physical processes are based on conservation of charge and mass complemented by the Butler-Volmer kinetics equation [23, 24]. Conservation equations of species and charge are derived from using porous electrode and concentrated solution theories and are solved over multiple phases to evaluate lithium concentration and electric potentials. We derive the continuum model equations using volume averaging techniques that are applicable to 
the entire cell with arbitrary 3D electrode configuration. The electrode and the electrolyte are considered as superimposed continua $[25,16,26]$, as shown in Fig. 1. This assumes that the microscopic features of the electrode are small compared to the overall domain and that their effect can be described by their statistical properties. The effect of nanoscale structures can be accounted by anisotropy in the electrode effective properties, such as permeability and conductivity. Our formulation allows for spatial and temporal variations in these properties which is critical for capability to model the evolution of the electrode structure over its life-time.

Let $L$ be the characteristic length of the averaged volume that is larger than the secondary particles i.e., $L>>r$ and $g(r)$ is a normalized weighting function that is monotonically decreasing over the distance $r$. The averaging function is defined as:

$$
4 \pi \int_{0}^{\infty} g(r) r^{2} d r=1
$$

Given this function, we can define local spatial averages of interest to construct the volume averaged equations. Consider a representative volume element $d V$ (Fig. 1) over which the quantities of interest are averaged as

$$
\langle\phi\rangle=\int_{V} \phi g(r) d V
$$

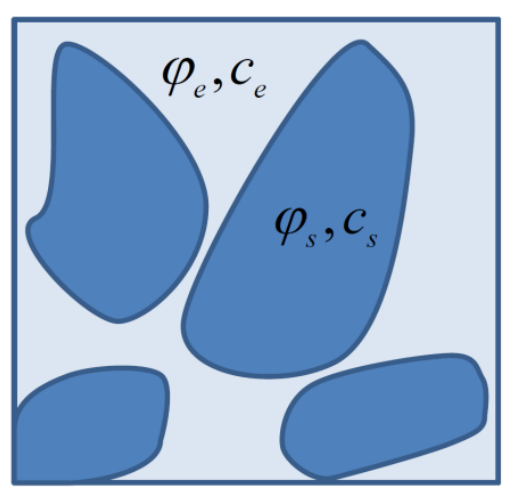

Fig. 1 Schematic of a representative volume 
Using these relations we can derive the conservation equations for each phase (gas, liquid and solid) averaged over a volume element that is larger than the primary particles and smaller than the device scale (here, the electrode thickness or the secondary particle diameter). Let $d V$ be the volume of representative element containing a solid phase and electrolyte phase.

For the quantity of interest $\psi$ the volume averages of the transient and spatial derivatives can be expressed in terms of corresponding derivatives of volume averages [27]

$$
\begin{aligned}
& \left\langle\frac{\partial}{\partial t}\right\rangle=\frac{\partial\langle\rangle}{\partial t} \frac{1}{V} \int_{A_{k}} \cdot n d A \\
& \langle\nabla\rangle=\nabla\langle\rangle+\frac{1}{V} \int_{A_{k}} n d A
\end{aligned}
$$

In the absence of convection in the electrolyte these equations can be rewritten as over an intrinsic volum2e average,

$$
\begin{aligned}
\left\langle\frac{\partial}{\partial t}\right\rangle & =\frac{\partial\langle\rangle}{\partial t} \\
\langle\nabla\rangle & =\nabla\langle\rangle
\end{aligned}
$$

where $\varepsilon$ is the volume fraction of the corresponding phase in averaging volume $d V$.

\subsection{Governing equations}

Concentrated solution theory [28] is used to model transport of ions in the electrolyte as the presence of individual ions could influence the net electric fields which can cause the properties to change. Using the volume averaging relationships Eq. (4) the conservation equation of lithiumion concentration $c_{e}$ within the electrolyte solution is given by

$$
\frac{\partial\left(\varepsilon_{e} c_{e}\right)}{\partial t}-\nabla \cdot\left(\varepsilon_{e} D_{e}^{e f f}\left(\varepsilon_{e}\right) \nabla c_{e}\right)=\frac{1-t_{+}^{0}}{F} j^{L i}
$$

where the effective diffusion coefficient is computed using the relation $D_{e}^{e f f}\left(\varepsilon_{e}\right)=D_{e} \varepsilon_{e}^{0.5}$. The 
last term of the equation represents the rate at which the ions are entering or leaving the electrolyte during intercalation/de-intercalation process at the secondary particle surfaces. The cation transference number $t_{+}^{0}$ indicates the efficiency of the intercalation process influencing the ion concentration in electrolyte [15].

Transport of $\mathrm{Li}$ in the solid phase occurs at a length scale different than the characteristic length of the problem. The current research assumes the electrode particles to be spherical in shape and the diffusion equation corresponding to solid phase transport is solved in spherical coordinates at every volume element. Analytical solutions are derived by using Duhamel superposition method. Diffusion lengths in spherical particles are used to compute solid phase surface concentration subjected to constant ion flux. The analytical solutions are used as closures for upscaling to continuum models and to evaluate the average solid phase surface concentrations. It should be mentioned however that the formulation presented in the current work can incorporate other closure terms, developed based on real electrode microstructures. Using again the volume averaging formulas in Eq. (4) we write the differential equations for solid phase concentration as,

$$
\frac{\partial\left(\varepsilon_{s} c_{s, a v g}\right)}{\partial t}-\nabla \cdot\left(\varepsilon_{s} D_{s}^{e f f}\left(\varepsilon_{s}\right) \nabla c_{s, a v g}\right)=-\frac{j^{L i}}{F}
$$

where $D_{s}^{e f f}\left(\varepsilon_{s}\right)=D_{s} \varepsilon_{s}^{0.5}$ and the closure relation given by,

$$
c_{s}=c_{s, a v g}+\frac{j^{L i} R_{s}}{5 a_{s} F D_{s}}
$$

Under the assumption of electroneutrality and absence of large electric fields in the electrolyte phase, accumulation of charge and discharge at the electric double layer is neglected and the conservation of charge happens instantaneously. The volume averaging [27, 23], the 
conservation of charge, and the Ohm's law (that relates the gradient of electric potential to the ionic current density in the electrolyte) results in the following equation

$$
\nabla \cdot\left(\varepsilon_{e} \kappa^{e f f}\left(\varepsilon_{e}\right) \nabla \phi_{e}\right)+\nabla \cdot\left(\varepsilon_{e} \kappa_{D}^{e f f}\left(\varepsilon_{e}\right) \nabla \ln c_{e}\right)=-j^{L i}
$$

where $\kappa^{\text {eff }}\left(\varepsilon_{e}\right)$ and $\kappa_{D}^{\text {eff }}\left(\varepsilon_{e}\right)$ are ionic and diffusive conductivity given by,

$$
\left\{\begin{array}{l}
\kappa^{e f f}\left(\varepsilon_{e}\right)=\kappa \varepsilon_{e}^{0.5} \\
\kappa_{D}^{e f f}\left(\varepsilon_{e}\right)=\frac{2 R T \kappa \varepsilon_{e}^{0.5}}{F}\left(t_{+}^{0}-1\right)\left(1+\frac{d \ln f_{+}}{d \ln c_{e}}\right)
\end{array}\right.
$$

The volume averaged equation for conservation of charge in solid phase is given by

$$
\nabla \cdot\left(\varepsilon_{s} \sigma^{e f f}\left(\varepsilon_{s}\right) \nabla \phi_{s}\right)=j^{L i}
$$

where $\sigma^{\text {eff }}\left(\varepsilon_{s}\right)=\sigma$ is the effective solid-phase conductivity.

The Butler-Volmer kinetics equation describes the total transfer current at the electrode / electrolyte interface corresponding to reaction $j$

$$
i_{n j}=i_{0}\left[\exp \left(\frac{\alpha_{a j} F}{R T} \eta_{j}\right)-\exp \left(-\frac{\alpha_{c j} F}{R T} \eta_{j}\right)\right]
$$

The coefficients $\alpha_{c j}$ and $\alpha_{a j}$ represent to the fraction of the applied potential that promotes cathodic and anodic reaction correspondingly. The surface overpotential drives the system away from equilibrium and is expressed as $\eta_{j}=\varphi_{s}-\varphi_{e}-U_{j, r e f}$, with $U_{j, r e f}$ denoting the equilibrium (open circuit) potential.

The exchange current density is defined as a function of rate constants for anodic and cathodic reactions and species concentrations 


$$
i_{0}=k\left(c_{e}\right)^{\alpha_{a j}}\left(c_{s, \max }-c_{s}\right)^{\alpha_{a j}}\left(c_{s}\right)^{\alpha_{c j}}
$$

and the pore-wall flux of lithium ions is related to reaction current densities as

$$
j^{L i}=\left\{\begin{array}{lr}
a_{s 1} i_{n 1} & \text { cathode } \\
0 & \text { separator } \\
a_{s 2} i_{n 2} & \text { anode }
\end{array}\right.
$$

,where specific interfacial area is given by $a_{s}=3 \varepsilon_{s} / R_{s}$.

\section{Computational methodology and implementation}

The initial value problem for a differential algebraic equations (DAE) system representing the conservation equations of mass and charge of a battery with dependent variables as

$\psi=\left\{c_{s}, c_{e}, \varphi_{s}, \varphi_{e}, j^{L i}\right\}$ can be written in the form,

$$
F(t, \psi, \dot{\psi})=0
$$

with given initial conditions $\psi(0)=\psi_{0}$ and $\dot{\psi}(0)=\dot{\psi}_{0}$. It should be pointed out that in this formulation the ionic pore-wall flux is the part of the solution at each time step rather than being lagged from the previous time step. In this system of equations the potential variables are the algebraic constraints making it into index 1 DAEs. Numerically time integrating this system is not possible unless consistent initial solutions for the algebraic constraints as well as the transient terms of the differential equations are imposed at initial time $t_{0}$. Hence for computing solution of these DAE's an essential pre-requisite is that the pair of vectors $\psi_{0}$ and $\dot{\psi}_{0}$ are both initialized to satisfy the Eq. (14). In the following sections we describe the techniques used to initialize the solutions and the solver strategy used for adaptive time integration of the resulting nonlinear equations. 
Inconsistent initial conditions can cause numerical solution methods to fail for an index-1 system of DAEs. Inconsistent initializations can be caused by alternating boundary conditions characteristic of application of a vehicle drive cycle profile where series of charge and discharge pulses can be encountered. Under a galvanostatic discharge a constant flux condition and a reference potential are imposed on the non-adjacent boundaries. At any instant $t$, the assumed constant initial solution profiles are inconsistent with the boundary conditions. It is not trivial to determine the consistent initial solution by the trial and error method. To calculate the correct initial conditions, the system of equations is recast into steady state equations for $t=t_{0}$ by change of variables with $\dot{c}=\tilde{c}$ and $c(x, y, z)=c^{\text {const }}$.

$$
\begin{aligned}
& 0=f\left(0, \tilde{c}, c^{c o n s t}, \phi, j^{L i}\right) \\
& 0=g\left(0, c^{\text {const }}, \phi, j^{L i}\right)
\end{aligned}
$$

where $c, \phi$, and $j^{L i}$ are vectors of differentiable and algebraic variables. The solution of this non-linear system of equations (we use Newton-Krylov method [29]) will be consistent with the boundary conditions. Such re-initialization technique should be used for step changes in the current boundary conditions which commonly occur during the pulse- or drive-cycle testing of batteries.

For the time integration scheme we use the variable order, variable coefficient backward differentiation formula (BDF) in the fixed leading coefficient form that is implemented in the IDA module of the Suite of Nonlinear and Differential/Algebraic Equation Solvers (SUNDIALS) [30]. The order ranges from 1 to 5 of the BDF given by the formula,

$$
\sum_{i=0}^{q} \alpha_{n, i} \psi_{n-i}=h_{n} \dot{\psi}_{n}
$$


where $\psi_{n}$ and $\dot{\psi}_{n}$ are the computed approximations to $\psi\left(t_{n}\right)$ and $\dot{\psi}\left(t_{n}\right)$, respectively, and the step size is given by $h_{n}=t_{n}-t_{n-1}$. The coefficients $\alpha_{n, i}$ are uniquely determined by the order $q$, and the recent history of the step sizes. Evaluating Eq. (14) at $t=t_{n}$ and using Eq. (17) to replace $\dot{\psi}$ leads to a nonlinear algebraic system to be solved at each step:

$$
F\left(t_{n}, \psi_{n}, \frac{1}{h_{n}} \sum_{i=0}^{q} \alpha_{n, i} \psi_{n-i}\right)=0
$$

Newton iteration applied to the nonlinear DAEs generates a sequence of approximations $\psi_{k}$ to the exact solution $\psi^{*}$, where $\psi_{k+1}=\psi_{k}+s_{k}$ and the Newton step $s_{k}$ is the solution to the system of linear equations

$$
F^{\prime}\left(\psi_{k}\right) s_{k}=-F\left(\psi_{k}\right)
$$

where $F^{\prime}$ is the Jacobian of $F$ evaluated at $\psi_{k}$. The Jacobian $J$ is the matrix of first order partial derivatives of the system

$$
J=\frac{\partial F}{\partial \psi}+\alpha \frac{\partial F}{\partial \dot{\psi}}
$$

, where $\alpha=\alpha_{n, 0} / h_{n}$. We use Inexact Newton iteration with scaled preconditioned Generalized Minimum Residual Method (GMRES) [31] as the linear solver for solving non-symmetric nonpositive definite systems. The method is relatively expensive and takes many iterations per Jacobian solve. In order to reduce the number of GMRES iterations we improve the condition number of the Jacobian matrix by preconditioning the system. Left preconditioning is utilized and for the preconditioner solve we use the Algebraic multigrid (Trilinos ML) V-cycle solver with a coarse grid solver [32]. The computational methodology described above was implemented 
in Advanced Multi-Physics for Electrical and Renewable Energy Storage (AMPERES) software based on AMP framework [38].

\section{Results and discussion}

\subsection{Application to planar geometry}

In this example we demonstrate the capability to perform a coupled simulation on the $3 \mathrm{D}$ domain with uniform material properties validated against experiments. For this, we present a simple 3D cell sandwich (Fig. 2(a)) with homogenized uniform material properties for the anode and cathode domains and we employ constant void fraction and tortuosity values assuming a uniform porosity across the material. For validation we choose experimental data from Doyle et.al. [15].

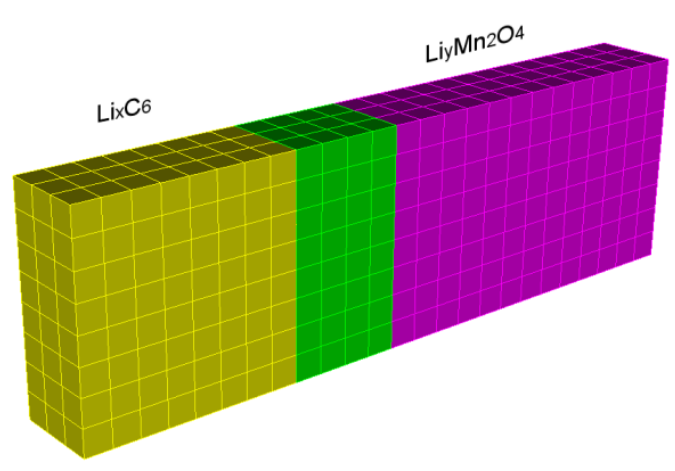

(a)

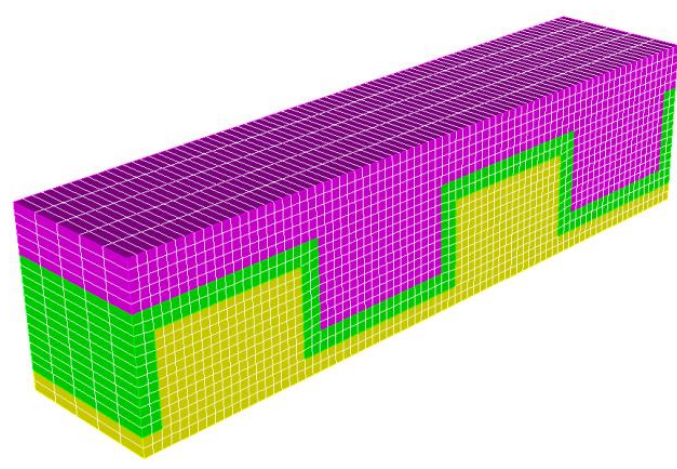

(b)

Fig. 2 (a) Planar cell sandwich configuration (b) 3D interdigitated electrode geometry

The results in Figs. 3, 4 are shown for a Lithium-polymer cell [15] consisting of carbon anode and a LiMn2O4 cathode with LiPF6 2:1 EC:DMC / p(VdF-HFP) polymer electrolyte. The geometric and electrochemical parameters were taken from $[15,16]$ and are given in Table 1 . The current collectors were represented by boundary conditions consisting of a uniform current flux on one end and reference potential on the other end of the cell sandwich. Current densities corresponding to a specific C-rate of galvanostatic discharge were applied. Figure 3 shows 
concentration profiles of the solid phase matrix in the cell sandwich at different times along the discharge. We can see that early in the discharge the concentration gradients develop near to the electrode/separator interface. It can be seen that the gradients disappear and the concentration drops uniformly across the negative electrode as the discharge progresses. In the positive electrode however steep gradients develop indicating that poor utilization of electrode material close to current collector. This internal phenomenon is more pronounced at higher $\mathrm{C}$ rates and can be clearly seen in Fig. 3(b). The predictions of discharge curves show good agreement with the experimental data as can be seen in Fig. 4(a) where the lines represent the results of simulation and the markers show the experimental data.

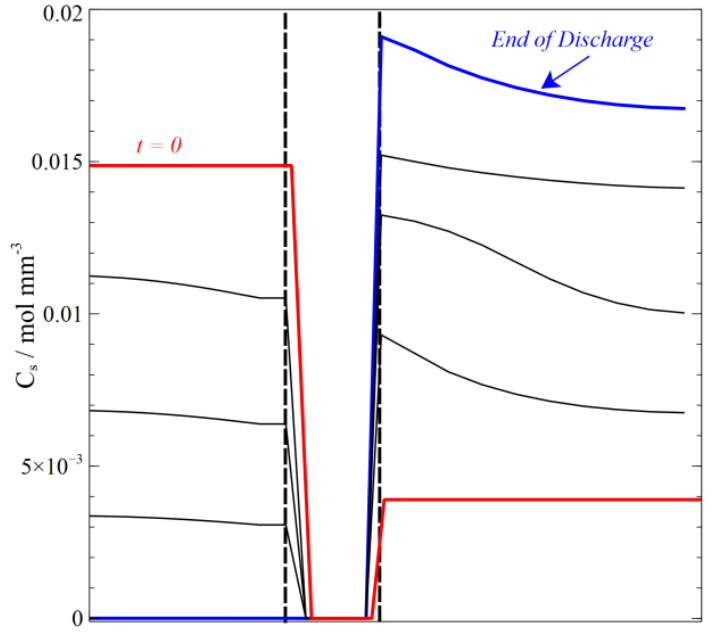

Distance

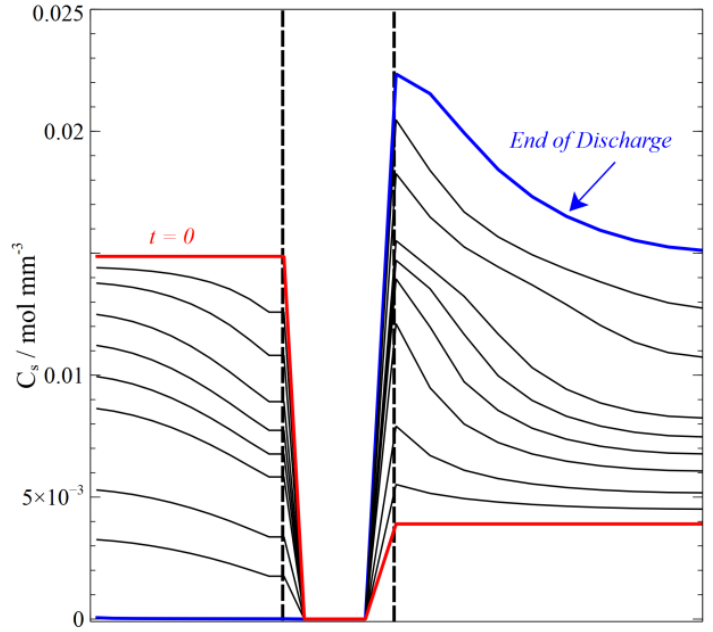

Distance

(a)

(b)

Fig. 3. Simulated solid phase lithium concentration profiles in cell sandwich under (a) 1C and (b) 5C discharge current. Vertical dashed lines indicate separator region.

The stability and robustness of the numerical technique for the 3D cell sandwich at very high discharge current depends on capturing the concentration gradients across the domains. At these high rates, initially the particles near the separator boundary experience the reactions and later on the reaction front quickly propagates across the electrode. The concentration gradients within the particles scale with the current and at small discharge currents the surface concentration and 
average concentration in a lithium particle can be approximated using linear relationship, Eq.(7), which holds for spherical particles where diffusion length [33] is $R_{s} / 5$. Such formulation however implies that the concentration immediately reaches its steady state level once the current is applied, i.e. the difference between the average and surface concentration is $j^{L i} R_{s} / 5 a_{s} F D_{s}$ at $t=0$. While the approximation in Eq.(7) works well at longer times, when the diffusion layer builds up to its steady state, it becomes invalid at short times when the concentration gradient starts from zero. This is especially important in cases when high current densities are applied and discharge happens rather quickly so the initial transient needs to be captured correctly. We use the correction to the Eq.(7) proposed in [33] which utilizes the exponential increase in concentration at short times and serves as better approximation to the Duhamel's solution for spherical particles

$$
c_{s}(t)=c_{s, a v g}(t)+\frac{j^{L i} l_{s}}{a_{s} F D_{s}}\left[1-\exp \left(-4 \sqrt{D_{s} t} / 3 l_{s}\right)\right]
$$

where $l_{s}=R_{s} / 5$ for spherical geometry. With this correction we were able to push the discharge currents up to $100 \mathrm{C}$ without any degeneracy in the computational process. This capability is demonstrated in Fig. 4(b) where we show the computed discharge profiles for various $\mathrm{C}$ rates. 


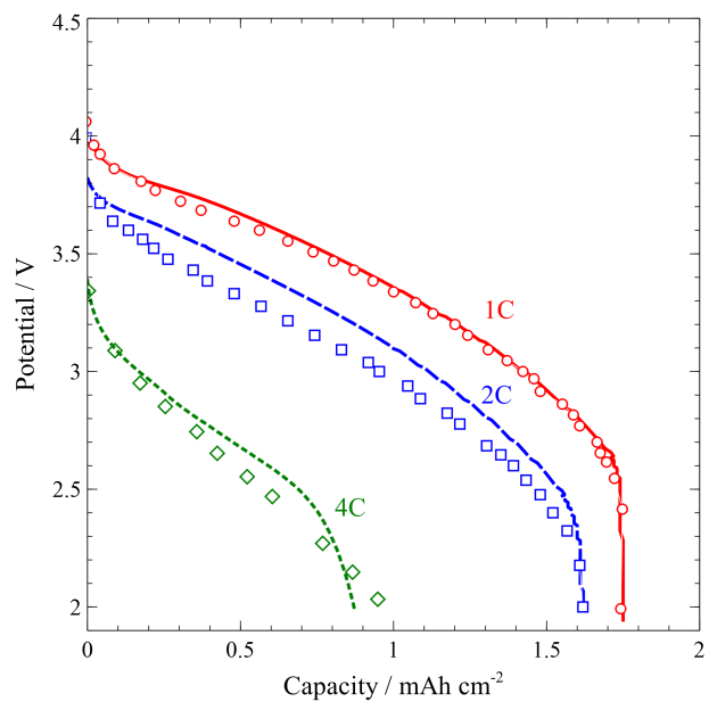

(a)

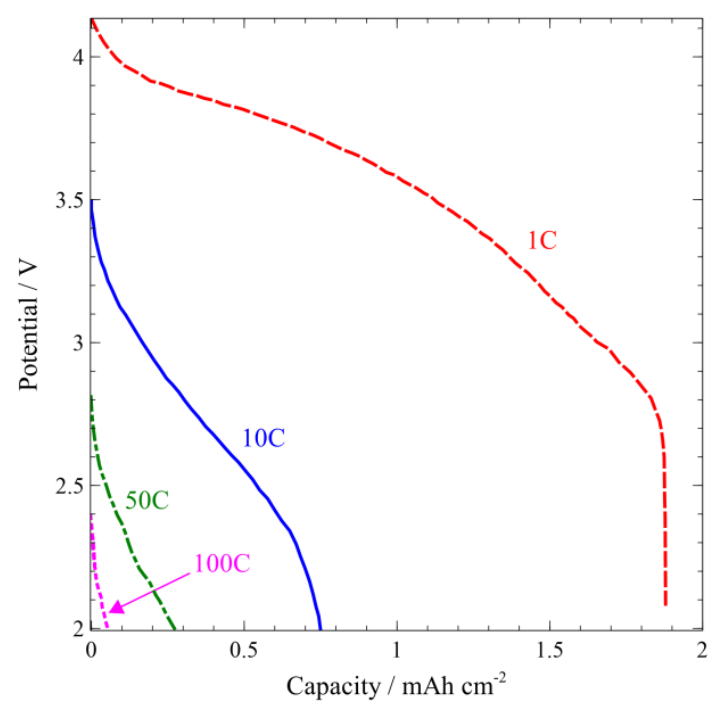

(b)

Fig.4 Modeling galvanostatic discharge of a cell under different current densities: (a) validation by experimental results [15]; (b) capability to model the behavior under very high discharge rates.

\subsection{Application to non-planar interdigitated geometry}

Due to limitations of conventional 2D thin-film batteries, 3D architectures are being synthesized to exploit the extra dimension for effective increase in area/volume ratio. Increased surface area should result in increase in power density with the same volume of active material. While being far from commercialization such 3D designs are being actively explored within the research community [34-36]. A comprehensive review can be found in [34]. Among different designs (posts, scaffolds, etc.) the simplest arrangement to manufacture is represented by the interdigitated electrodes and this is the geometry chosen in the current work to demonstrate the capability of the $3 \mathrm{D}$ volume averaged computational model. The example of the trenched electrodes periodic structure with corresponding finite element mesh is shown in Fig. 2(b). The micro-battery in Fig. 2(b) was $1.85 \mathrm{~mm}$ long and $0.4 \mathrm{~mm}$ wide.

To evaluate the performance of the 3D designs in comparison to the conventional planar $2 \mathrm{D}$ batteries, we maintain a constant volume of electrodes in all configurations to ensure same specific energy density. In other words we keep the height and width of the cell sandwich 
constant and vary the thickness of the electrodes. In this construction process, we reduce the thickness of the electrodes in planar configuration and introduce the reduced volume in the form of trenches with equal height on either electrode. Equal height is required to accommodate the constant thickness of the separator that is spanning across the trenches. The three configurations that are used in the performance study are shown in Fig. 5. There is a factor of two difference between the shallow (Fig. 5(b)) and deep trench depth (Fig. 5(c)). With this we maintained constant energy density of the three batteries but increased the surface area that is exposed to initial reaction fronts. As the energy density of the system is maintained constant, these three configurations can be subjected to same boundary conditions of constant current discharge at various C-rates. The same cell parameters as those listed in the previous example (Table 1) are used here. The constant volume of the active material is based on the electrode thicknesses from the planar geometry listed in Table 1.

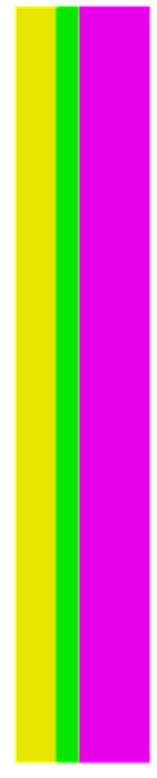

(a)

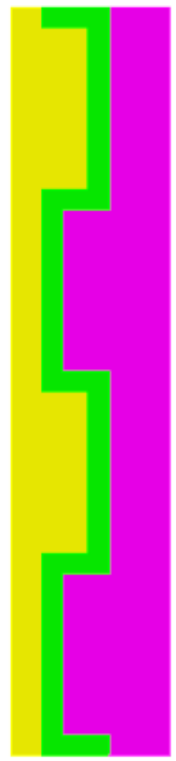

(b)

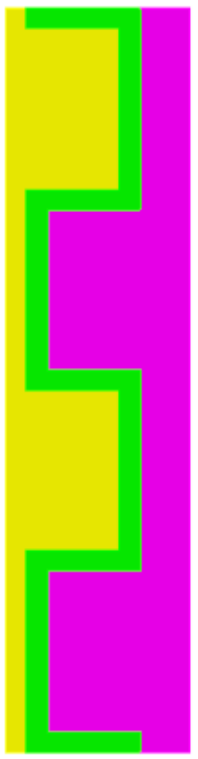

(c)

Fig. 5 Three cell sandwich geometries compared in simulations: (a) planar; (b) shallow trench; (c) deep trench (depth is $2 \mathrm{x}$ of shallow trench). 
In addition to maintaining the constant volume of active material, the material balance between the positive and negative electrodes needs to be maintained as well which implies preserving the equal number of trenches on the both sides of the cell sandwich. An unintended consequence of this is that we forgo the symmetry of the system. As the main purpose of this study is comparative analysis of the three geometries with increasing surface area, we adhere to the un-symmetric configurations despite the fact that this results in unsymmetrical solution. In Fig. 6 we show the comparison of the solid phase concentration profiles among three configurations at the end of $1 \mathrm{C}$ discharge. The skewness in the concentration distribution can be seen which is the result of un-symmetric design discussed above. A small gradient in the concentration distribution can be observed in planar geometry (Fig. 6(a)). Same gradients still can be seen in the shallow trench configuration (Fig. 6(b)) while the distribution becomes more uniform as the trenches are made deeper (Fig. 6(c)). Higher Li utilization can also be observed with concentrations being generally higher in interdigitated electrodes approaching the maximum stoichiometric solid concentration in the positive electrode in the case of deep trenches (Fig. $6(\mathrm{c}))$. 


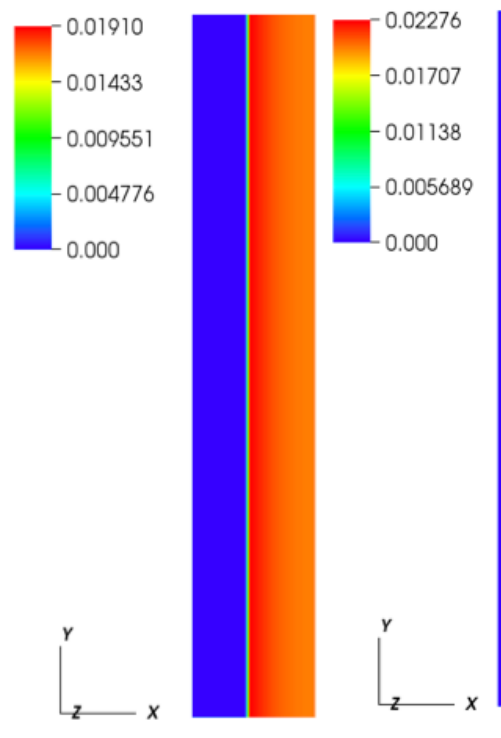

(a)

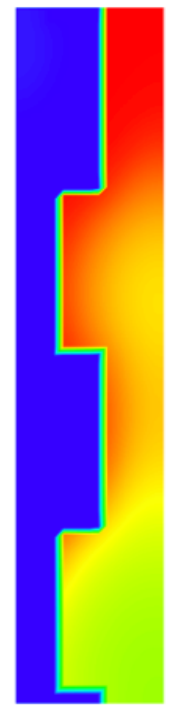

(b)

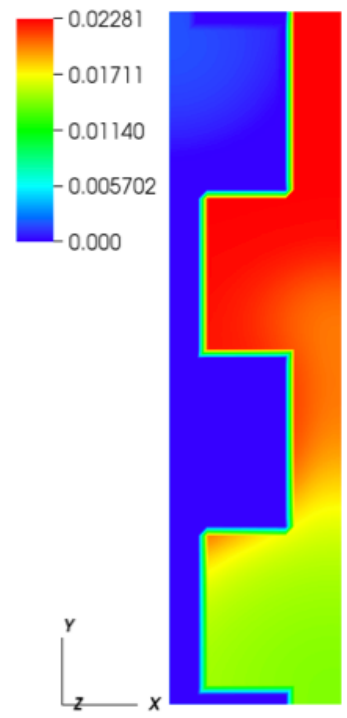

(c)

Fig. 6 Lithium concentration in solid phase $\left(\mathrm{mol} \mathrm{mm}^{-3}\right)$ at the end of $1 \mathrm{C}$ discharge: (a) planar geometry; (b) shallow trench; (c) deep trench (depth is $2 \mathrm{x}$ of shallow trench).

Lithium content in solid phase at the end of $5 \mathrm{C}$ discharge is shown in Fig. 7. The active material is utilized primarily in the region near to the separator in planar configuration (Fig. 7(a)) with significant concentration gradient developed across the positive electrode. Better material utilization (lithium concentration in solid electrodes) can be seen in interdigitated designs in Fig. 7 (b, c). 


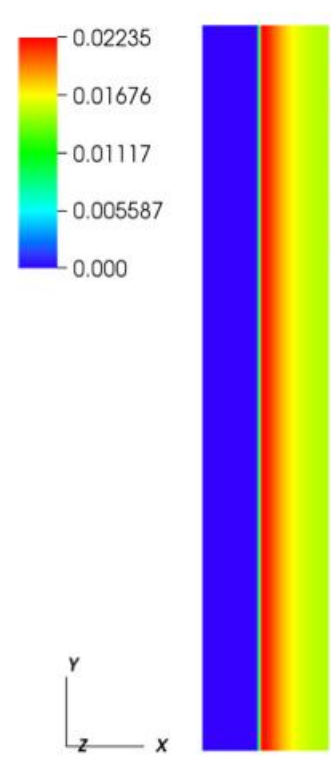

(a)

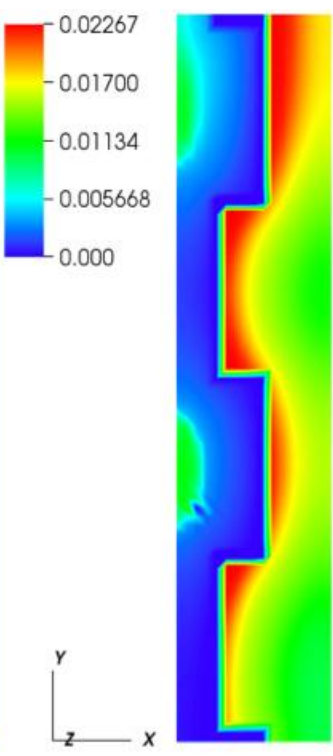

(b)

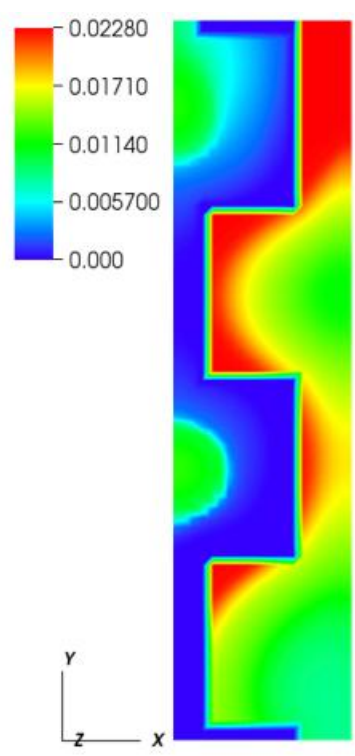

(c)

Fig. 7 Lithium concentration in solid phase $\left(\mathrm{mol} \mathrm{mm}^{-3}\right)$ at the end of $5 \mathrm{C}$ discharge: (a) planar geometry; (b) shallow trench; (c) deep trench (depth is $2 \mathrm{x}$ of shallow trench).

Better utilization of the electrode material results in higher discharge capacity for interdigitated designs of cell sandwich as shown in Fig. 8(a). As the depth of the trenches increases so does the cell capacity, while the specific energy density remains the same as the total volume of the electrode materials is kept constant. The power/energy characteristics of an energy storage device are best explored with Ragone plots traditionally represented in volumetric energy and power coordinates. The power and energy densities corresponding to the three discharge currents in Fig. 8(a) were used to construct Ragone plots for different cell designs as shown in Fig. 8(b). The curves in Fig. 8(b) suggest that for a given energy density more power can be extracted when deviating from planar cell configuration to three dimensional architectures. The gain in power under highest applied current due to introduction of deep trenches is approximately a factor of 1.5 compared to planar electrode arrangement which is close to the factor by which the electrode surface area increased between the two geometries. In addition change in the slope of the energy density drop can be noticed when the electrode surface area is increased via 
introduction of trenches.

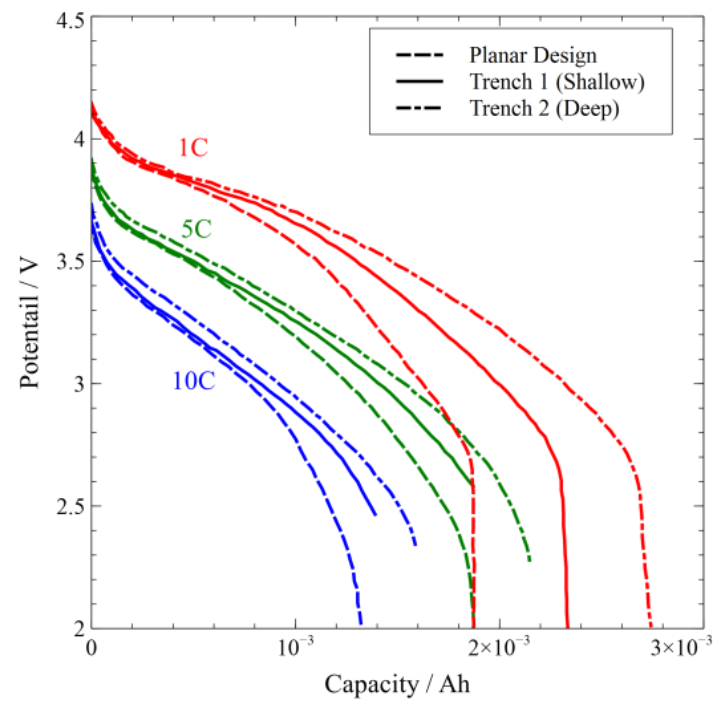

(a)

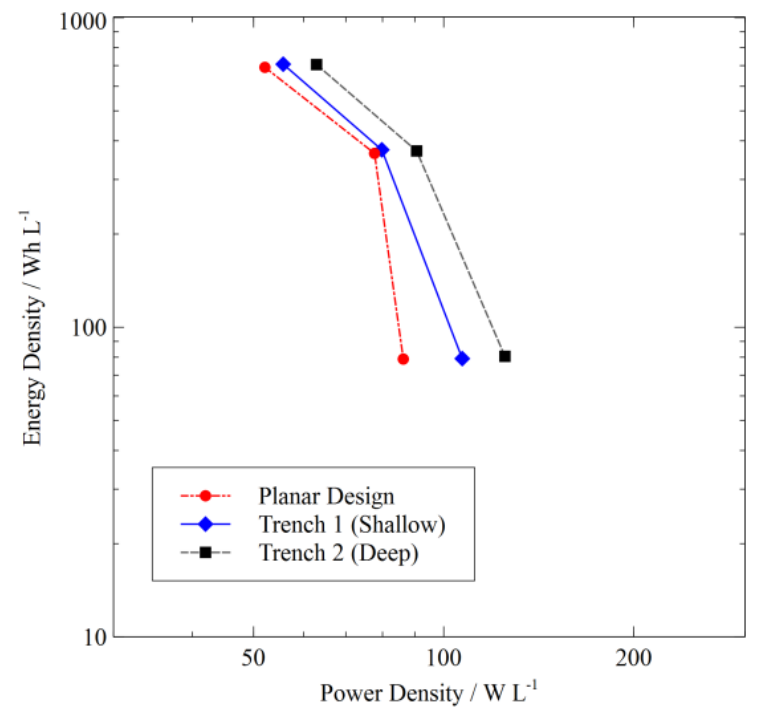

(b)

Fig. 8 Performance characteristics of different cell designs: (a) discharge profiles; (b) Ragone plot

\section{Conclusions}

A 3-dimensional computational model for electrochemical performance of batteries has been developed using a single domain volume-averaged approach. Such formulation eliminates the need for placement of boundary conditions at the interfaces for mass and charge transport. The pore-wall ion flux is included as a part of the solution vector of the DAE system at each time step. This distinguishes the proposed method from the currently available computational models for intercalation based batteries, where this variable is lagged from the previous time step. Appropriate initialization of variables which follows abrupt changes in boundary conditions has been utilized in the current approach together with corrected formulation of the surface concentration as better approximation to the exact solution of the solid phase diffusion problem. The latter provides capability to compute charge/discharge profiles under extremely high currents when the steady state assumption for solid state concentration is not valid.

The proposed model was validated by experimental result from the literature [15] and was 
applied to investigate benefits of 3-dimensional arrangements of the electrodes inside the cell.

The interdigitated design was found to increase extractable capacity from the cell compared to the planar electrode arrangement with the same energy density. Finally, due to better electrode material utilization, the power density of the cell increased with increase in electrode trench depth. This confirms that exploring 3D architectures for cells with higher power characteristics is a promising direction. The developed model is a suitable tool for investigation of such systems.

\section{Acknowledgment}

This research at Oak Ridge National Laboratory, managed by UT-Battelle, LLC, for the U.S. Department of Energy under contract DE-AC05-00OR22725, was sponsored by the Vehicle Technologies Program for the Office of Energy Efficiency and Renewable Energy.

\section{References}

[1] S. Allu, B.V. Asokan, W.A. Shelton, B. Philip, S. Pannala, A generalized multidimensional mathematical model for charging and discharging processes in a supercapacitor, Journal of Power Sources 256 (2014) 369-382.

[2] B. Kang, G. Ceder, Battery materials for ultrafast charging and discharging, Nature 458 (7235) (2009) 190-193.

[3] T.S. Arthur, D.J. Bates, N. Cirigliano, D.C. Johnson, P. Malati, J.M. Mosby, E. Perre, M. T. Rawls, A.L. Prieto, B. Dunn, Three-dimensional electrodes and battery architectures, Mrs Bulletin 36 (07) (2011) 523-531.

[4] J. Baxter, Z. Bian, G. Chen, D. Danielson, M.S. Dresselhaus, A. G. Fedorov, T.S. Fisher, C.W. Jones, E. Maginn, U. Kortshagen, et al., Nanoscale design to enable the revolution in renewable energy, Energy \& Environmental Science 2 (6) (2009) $559-588$. 
[5] D.R. Rolison, J.W. Long, J.C. Lytle, A.E. Fischer, C.P. Rhodes, T.M. McEvoy, M. E. Bourg, A.M. Lubers, Multifunctional 3d nanoarchitectures for energy storage and conversion, Chemical Society Reviews 38 (1) (2009) 226-252.

[6] G. Ceder, M. Doyle, P. Arora, Y. Fuentes, Computational modeling and simulation for rechargeable batteries, MRS bulletin 27 (08) (2002) 619-623.

[7] R.W. Hart, H.S. White, B. Dunn, D.R. Rolison, 3-d microbatteries, Electrochemistry Communications 5 (2) (2003) 120-123.

[8] N. Cirigliano, G. Sun, D. Membreno, P. Malati, C. Kim, B. Dunn, 3d architectured anodes for lithium-ion microbatteries with large areal capacity, Energy Technology 2 (4) (2014) 362-369.

[9] P. Arora, R. E. White, M. Doyle, Capacity fade mechanisms and side reactions in lithium-ion batteries, Journal of the Electrochemical Society 145 (10) (1998) $3647-3667$

[10] P. Arora, M. Doyle, R. E. White, Mathematical modeling of the lithium deposition overcharge reaction in lithium-ion batteries using carbon-based negative electrodes, Journal of The Electrochemical Society 146 (10) (1999) $3543-3553$.

[11] P. Arora, M. Doyle, A.S. Gozdz, R.E. White, J. Newman, Comparison between computer simulations and experimental data for high-rate discharges of plastic lithium-ion batteries, Journal of Power Sources 88 (2) (2000) 219-231.

[12] S. Santhanagopalan, Q. Guo, P. Ramadass, R.E. White, Review of models for predicting the cycling performance of lithium ion batteries, Journal of Power Sources 156 (2) (2006) 620-628.

[13] K. Kumaresan, G. Sikha, R.E. White, Thermal model for a li-ion cell, Journal of 
the Electrochemical Society 155 (2) (2008) A164-A171.

[14] B.S. Haran, B.N. Popov, R.E. White, Determination of the hydrogen diffusion coefficient in metal hydrides by impedance spectroscopy, Journal of Power Sources 75 (1) (1998) 56-63.

[15] M. Doyle, J. Newman, A.S. Gozdz, C.N. Schmutz, and J.M. Tarascon, Comparison of modeling predictions with experimental data from plastic lithium ion cells. Journal of the Electrochemical Society, 143(6) (1996) 1890-1903.

[16] C. Wang, W. Gu, B. Liaw, Micro-macroscopic coupled modeling of batteries and fuel cells I. Model development, Journal of the Electrochemical Society 145 (10) (1998) $3407-3417$.

[17] Q. Zhang, R. E. White, Comparison of approximate solution methods for the solid phase diffusion equation in a porous electrode model, Journal of Power Sources 165 (2) (2007) 880-886.

[18] R.E. García, Y.-M. Chiang, Spatially resolved modeling of microstructurally complex battery architectures, Journal of the Electrochemical Society 154 (9) (2007) A856-A864.

[19] U.S. Kim, C.B. Shin, C.-S. Kim, Modeling for the scale-up of a lithium-ion polymer battery, Journal of Power Sources 189 (1) (2009) 841-846.

[20] M. Tang, P. Albertus, J. Newman, Two-dimensional modeling of lithium deposition during cell charging, Journal of the Electrochemical Society 156 (5) (2009) A390A399.

[21] S. Allu, S. Kalnaus, W. Elwasif, S. Simunovic, J. A. Turner, S. Pannala, A new open computational framework for highly-resolved coupled three-dimensional multiphysics simulations of li-ion cells, Journal of Power Sources 246 (2014) 876- 
886.

[22] T.R. Ferguson, M.Z. Bazant, Nonequilibrium thermodynamics of porous electrodes, Journal of The Electrochemical Society 159 (12) (2012) A1967-A1985.

[23] P. De Vidts, R.E. White, Governing equations for transport in porous electrodes, Journal of the Electrochemical Society 144 (4) (1997) 1343-1353.

[24] W. Gu, C. Wang, S. Li, M. Geng, B. Liaw, Modeling discharge and charge characteristics of nickel-metal hydride batteries, Electrochimica Acta 44 (25) (1999) 4525-4541.

[25] T.B. Anderson, R. Jackson, Fluid mechanical description of fluidized beds. Equations of motion, Industrial \& Engineering Chemistry Fundamentals 6 (4) (1967) 527-539.

[26] S. Pannala, Computational Gas-Solids Flows and Reacting Systems: Theory, Methods and Practice, IGI Global, 2010.

[27] J.C. Slattery, Momentum, energy, and mass transfer in continua, McGraw-Hill New York, 1972.

[28] J. Newman, Transport properties of concentrated solutions, Electric Power Research Inst. Battery Conference, Palo Alto, CA Nov. 301983

[29] C.T. Kelley, Solving nonlinear equations with Newton's method, SIAM Book Series Fundamentals of Algorithms, 2003 ISBN: 978-0-89871-546-0, 100 pp.

[30] C. Hindmarsh, P.N. Brown, K.E. Grant, S.L. Lee, R. Serban, D.E. Shumaker, C. S. Woodward, Sundials: Suite of nonlinear and differential/algebraic equation solvers, ACM Transactions on Mathematical Software (TOMS) 31 (3) (2005) 363-396.

[31] Y. Saad, M.H. Schultz, Gmres: A generalized minimal residual algorithm for solving 
nonsymmetric linear systems, SIAM Journal on scientific and statistical computing 7 (3) (1986) 856-869.

[32] M.A. Heroux, R.A. Bartlett, V.E. Howle, R.J. Hoekstra, J.J. Hu, T.G. Kolda, R.B. Lehoucq, K.R. Long, R.P. Pawlowski, E.T. Phipps, et al., An overview of the trilinos project, ACM Transactions on Mathematical Software (TOMS) 31 (3) (2005) 397423.

[33] C. Wang, V. Srinivasan, Computational battery dynamics (CBD) electrochemical/thermal coupled modeling and multi-scale modeling, Journal of Power Sources 110 (2) (2002) 364-376.

[34] J.W. Long, B. Dunn, D.R. Rolison, H.S. White, Three-dimensional battery architectures, Chem. Rev. 104 (2004), 4463-4492.

[35] L. Baggetto, R.A.H. Niessen, F. Roozeboom, P.H.L. Notten, High energy density all solid state batteries: a challenging concept towards 3D integration, Advanced Functional Materials 18 (2008) 1057-1066.

[36] J.H. Pikul, H.G. Zhang, J. Cho, P.V. Braun, W.P. King, High-power lithium ion microbatteries from interdigitated three-dimensional bicontinuous nanoporous electrodes, Nature Communications 4:1732 (2013).

[37] V. Zadin, H. Kasemagi, A. Aabloo, D. Brandell, Modeling electrode material utilization in the trench model 3D-microbattery by finite element analysis, J. Power Sources 195 (2010) 6218-6224.

[38] B. Philip, M.A. Berrill, S. Allu, S.P. Hamilton, R.S. Sampath, K.T. Clarno and G.A. Dilts. A parallel multi-domain solution methodology applied to nonlinear thermal transport problems in nuclear fuel pins, Journal of Computational Physics, 286 (2015), 143-171. 
Table 1. Parameters used for electro-chemical model $([15,16])$

\begin{tabular}{|c|c|c|c|c|}
\hline Symbol & Units & Anode (Carbon) & Separator & Cathode $\left(\mathrm{LiMn}_{2} \mathrm{O}_{4}\right)$ \\
\hline$\overline{L L}$ & $\mu m$ & 100 & $\overline{52}$ & 174 \\
\hline$\varepsilon_{s}$ & - & 0.471 & - & 0.297 \\
\hline$\varepsilon_{e}$ & - & 0.503 & 0.724 & 0.630 \\
\hline$\varepsilon_{f}$ & - & 0.026 & - & 0.073 \\
\hline$c_{s}^{\max }$ & $\mathrm{mol} / \mathrm{m}^{3}$ & 26390 & - & 22860 \\
\hline$c_{s}^{0}$ & $\mathrm{~mol} / \mathrm{m}^{3}$ & 14870 & - & 3900 \\
\hline$c_{e}^{0}$ & $\mathrm{~mol} / \mathrm{m}^{3}$ & 2000 & & \\
\hline$D_{s}$ & $m^{2} / s$ & $3.9 \cdot 10^{-14}$ & - & $1.0 \cdot 10^{-13}$ \\
\hline$D_{e}$ & $m^{2} / s$ & $7.5 \cdot 10^{-11}$ & & \\
\hline$i_{0}$ & $A / m^{2}$ & 1.1 & - & 0.8 \\
\hline$\alpha_{a}$ & - & 0.5 & - & 0.5 \\
\hline$\alpha_{c}$ & - & 0.5 & - & 0.5 \\
\hline$t_{+}^{0}$ & - & 0.363 & & \\
\hline$\sigma$ & $S / m$ & 100 & - & 3.8 \\
\hline$R_{s}$ & $\mu m$ & 12.5 & - & 8.5 \\
\hline
\end{tabular}

